九州大学学術情報リポジトリ

Kyushu University Institutional Repository

\title{
Modeling of the rotation of polarization in polymers using an inhomogeneous birefringence mode l
}

Watanabe, Yukio

Mitsubishi Kasei Research Center

http://hdl. handle. net/2324/4493208

出版情報 : Journal of Applied Physics. 76 (7), pp.3994-4002, 1994-10-01. American Institute of Physics

バージョン :

権利関係：○ 1994 American Institute of Physics 


\section{Modeling of the rotation of polarization in polymers using an inhomogeneous birefringence model}

Cite as: Journal of Applied Physics 76, 3994 (1994); https://doi.org/10.1063/1.357345

Submitted: 07 February 1994 . Accepted: 20 June 1994 . Published Online: 04 June 1998

\section{Yukio Watanabe}

Challenge us.

What are your needs for periodic signal detection?

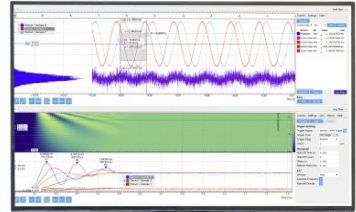

Zurich

Instruments 


\title{
Modeling of the rotation of polarization in polymers using an inhomogeneous birefringence model
}

\author{
Yukio Watanabe \\ Mitsubishi Kasei Research Center, Kamoshida 1000, Midoriku, Yokohama Kanagawa 227, Japan
}

(Received 7 February 1994; accepted for publication 20 June 1994)

\begin{abstract}
Polymers exhibiting a weak birefringence mostly posses optical properties which are predicted well by conventional models for birefringence. However, the rotation of polarization behavior was found to disagree with these models. This deviation has been overlooked in usual measurements characterizing birefringence in polymers. In order to accurately predict the rotation of polarization, we propose a model which includes a birefringence inhomogeneity. The model is shown to be related to the birefringence-induced optical activity. The model predictions are shown to agree well with experimental results using a parallel laser beam. Practical implications with respect to unusually high low-frequency noise of magneto-optical disks are discussed briefly.
\end{abstract}

\section{INTRODUCTION}

Recently, optical properties of random media have received considerable attention. Especially birefringence of polymers has been intensively discussed due to its practical importance in magneto-optical recording ${ }^{1-3}$ and liquid crystal displays. Although mathematical models for polarization and birefringence using classical crystal optics have been well established, a close examination of the polarization properties has only recently become possible.

Figure 1 shows the problem discussed in this article. After a linearly polarized beam passes through a plastic plate, it generally becomes elliptically polarized with its polarization being rotated. Here, the direction of polarization is defined as the direction where the laser power measured through the analyzer is at its maximum. This rotation of polarization even occurs in uniaxial and biaxial media and is correctly predicted by existing models. ${ }^{4}$ However, it accompanies elliptical polarization and is therefore not a pure rotation of polarization. Since the effect is typically small for a medium with a small retardation, this rotation of polarization has not received much attention.

Classical models for birefringence in polymer substrates have been based on the use of refraclive ellipsoids which are valid for single crystals. ${ }^{4}$ In most cases, these models have been successful in accounting for the optical properties of transparent plastics.

However, a close examination showed that the rotation of polarization of a transmitted beam was not in accordance with these models for optically inactive plastics such as polycarbonates, their derivatives, and epoxy. In Ref. 5 we have tentatively attributed these deviations to an inhomogeneity of birefringence and modeled it by using birefringent double layers.

In this article we generalize this idea and present a simple formula as a basis for birefringence modeling in plastics. We show that an inhomogeneous birefringence is equivalent to birefringence coexisting with optical rotatory power. We derive the formula using a birefringent multilayer model and later re-examine it using Maxwell equations. The model calculations compare favorably with experimental data. In addition, the effect of in-plane inhomogeneity is discussed to understand the low frequency modulation of magneto-optical disks.

\section{EXPERIMENT}

In this study, optical properties were measured in injection molded polycarbonate disks with a thickness of $1.2 \mathrm{~mm}$ used as a substrate for commercial magneto-optical disks because of their high purity, good homogeneity, and superior surface flatness. Figure 2 shows a block diagram of the experimental setup to measure the direction of the maximum electric power, i.e., the principal polarization direction. A randomly polarized He-Ne laser with a wavelength of 633 $\mathrm{nm}$ and a semiconductor laser with a wavelength of $820 \mathrm{~nm}$ were used as light beam sources. The beam was linearly polarized by a polarizer ( $\mathrm{PO}$ ), passed the sample, was modulated by a Faraday cell, passed through an analyzer (AO), and was finally detected by a photomultiplier. The output of the photomultiplier and the Faraday cell was measured by a lock-in amplifier.

The effective retardation of the $\mathrm{PO}$ and the $\mathrm{AO}$ was less than $1 \mathrm{~nm}$, and the effective retardation by the Faraday cell was about $1.5 \mathrm{~nm}$. When the power of the He-Ne laser through the PO was measured by rotating the PO, the beam was slightly elliptically polarized with the maximum power being about $5.7 \%$ higher than the minimum. The beam spot size on the sample was about $1 \mathrm{~mm}$ in diameter. The precision of rotation of the $\mathrm{PO}$ was $0.01^{\circ}$, and the precision of the sample positioning was better than $0.2 \mathrm{~mm}$. The laser power passing through the PO was about $2 \mathrm{~mW}$.

We used the following procedure to minimize the measurement error:

(1) The sample was moved away horizontally from the beam and the angle of the PO was set.

(2) The extinction angle was measured by rotating the $\mathrm{AO}(=\mathrm{AO}$ angle 1$)$.

(3) The sample was moved back into the beam.

(4) The angle was measured by rotating the $\mathrm{AO}$ (=AO angle 2) where the power through $\mathrm{AO}$ was the minimum.

(5) Steps (1)-(4) of the above procedure were repeated, and we defined a rotation angle of polarization $\Delta \theta$ as $\mathrm{AO}$ angle 2-AO angle 1 (Fig. 1). 


$$
1
$$

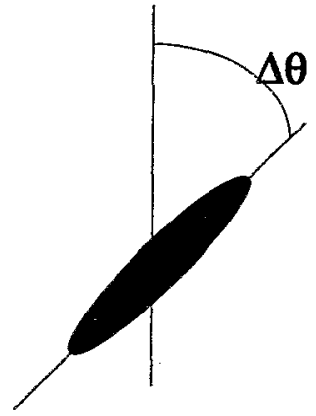

FIG. 1. Rotation of a principal direction of polarization through birefringence. Initial polarization is shown by an arrow. After passing through a birefringent medium, the beam is elliptically polarized and polarization rotates by an angle $\Delta \theta$. In plastics, the magnitude of $\Delta \theta$ is different from the uniaxial medium. Experimentally, $\Delta \theta=$ polarizer angle at the maximum electric field-analyzer angle at the maximum electric field.

The magnitude and the principal direction of retardation varied over the sample. The error caused by inaccurate positioning was $\sim 5 \%$ or $1-2 \mathrm{~nm}$ in retardation and $1^{\circ}-2^{\circ}$ in the principal direction of retardation. Although no change of retardation was observed due to possible heating by the laser beam, it changed approximately $5 \%$ per hour due to variation of humidity and room temperature. Therefore, care was taken for the condition of the room atmosphere, and all measurements were done in a dark room.

\section{MATHEMATICAL FORMULATION}

\section{A. Birefringent multilayer model}

First, the inhomogeneous birefringence is modeled using a birefringent multilayer and a Jones matrix formulation. Consider a plate which consists of $N$ layers where the optical properties of each layer are expressed by a refractive ellipsoid with principal axes $n 1 \neq n 2 \neq n 3$. For our purpose, we can neglect the change of direction of the ray. Therefore, the refractive index in each layer is expressed by a refractive ellipse which is obtained by intersecting the refractive ellipsoid with the plane perpendicular to the incident beam. Consequently, each layer is regarded as a uniaxial medium. We may use Jones matrices and vectors when the thickness of

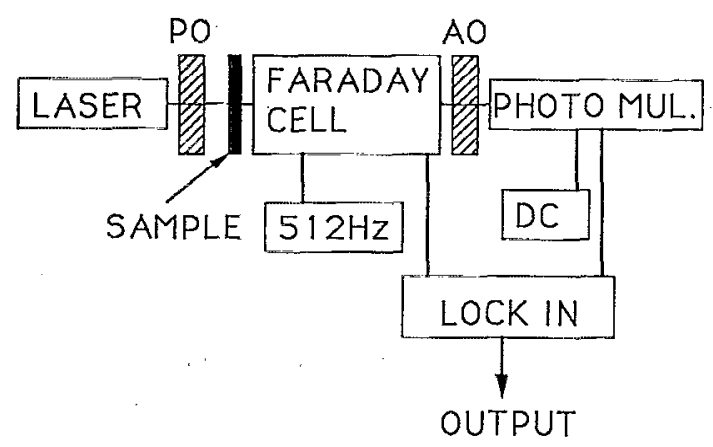

FIG. 2. Block diagram for a measuring of a change of a principal polarization dircetion through birefringence. $\mathrm{AO}, \mathrm{PO}, \mathrm{DC}$ denotes analyzer, polarizer, and dc power supply, respectively. the each layer $\mathscr{C}$ is much larger than the laser light wavelength $\lambda$. The Jones matrix $\mathbf{M}$ for birefringent $N$ layers is given by

$$
\begin{aligned}
& \mathbf{M}=\prod_{n=1}^{N} \mathbf{R}\left(\theta_{n}\right) \mathbf{P} \delta_{n} \mathbf{R}\left(-\theta_{n}\right), \\
& \mathbf{R}(\theta)=\left(\begin{array}{cc}
\cos \theta & -\sin \theta \\
\sin \theta & \cos \theta
\end{array}\right), \mathbf{P} \delta=\left(\begin{array}{cc}
\exp (i \delta) & 0 \\
0 & \exp (-i \delta)
\end{array}\right), \\
& \mathbf{E}=M \mathbf{E}_{0}=\left(\begin{array}{c}
E x \\
E y
\end{array}\right), \quad \mathbf{E}_{0}=\left(\begin{array}{c}
E_{0} x \\
E_{0} y
\end{array}\right),
\end{aligned}
$$

where $\theta_{n}$ and $\delta_{n}$ are the direction of the principal axis of birefringence and half the retardation in each layer, respectively. $\mathbf{P} \delta$ expresses the birefringence for a beam with its polarization parallel to a principal axis. $\mathbf{E}_{\mathbf{0}}$ and $\mathbf{E}$ are the electric field vectors of the incident and the transmitted beam, respectively. In the following, we introduce new representations of the Jones matrices and decompose $\mathbf{P} \delta$ into

$$
\begin{aligned}
& \mathbf{P} \delta=\cos \delta \mathbf{I}+i \sin \delta \mathbf{V} ; \quad \mathbf{I}=\left(\begin{array}{ll}
1 & 0 \\
0 & 1
\end{array}\right), \\
& \mathbf{V}=\left(\begin{array}{cc}
1 & 0 \\
0 & -1
\end{array}\right) .
\end{aligned}
$$

Similarly, birefringence with an arbitrary direction of the principal axis [Eq. (1) with $N=1$ ] is expressed by

$$
\begin{aligned}
& \mathbf{R}(\theta) \mathbf{P} \delta \mathbf{R}(-\theta)=\cos \delta \mathbf{I}+i \sin \delta \mathbf{S}(2 \theta), \\
& \mathbf{S}(\theta)=\mathbf{R}(\theta) \mathbf{V}=\left(\begin{array}{cc}
\cos \theta & \sin \theta \\
\sin \theta & -\cos \theta
\end{array}\right) .
\end{aligned}
$$

Equation (2) gives the well known results for uniaxial media. ${ }^{4}$ It should be noted that $\mathbf{M}$ is a unitary matrix. For a unitary matrix $\mathbf{U}(i, j)=x_{i j}+i y_{i j}\left(i, j=1,2, x_{i j}, y_{i j}\right.$ : real numbers) a constraint $\dagger \mathbf{U} \mathbf{U}=1$, where $\dagger \mathbf{U}(i, j)=x_{j i}-i y_{j i}$, reduces the number of free $x_{i j}$ and $y_{i j}$ to four. However, the choice of the phase $\beta$ is not physically meaningful in $\mathbf{U}^{\prime}(i, j)=e^{i \beta} \mathbf{U}(i, j)$. If we restrict this freedom, the number of free $x_{i j}$ and $y_{i j}$ reduces to three. Therefore, any unitary operator can be expressed by Eq. (3) below by choosing a suitable phase $\beta$, because Eq. (3) is a unitary operator with three free parameters. Thus, $\mathbf{M}$ can be written as

$$
\mathbf{M}=\cos \sigma \mathbf{R}(2 \alpha 0)+i \sin \sigma \mathbf{S}(2 \alpha) .
$$

Equation (3) is a generalization of Eq. (2) for a multilayer medium. A more physical derivation is given in Appendix A, and the rotation bias $\alpha 0$ was estimated to be of the order of $\sigma^{2}$ for a weak birefringence $\sigma<1$. The condition $\alpha 0=0$ corresponds to conventional birefringence. In Eq. (3) $\sigma$ and $\alpha$ are half of the effective retardation and the effective principal axis direction, respectively, if the birefringent $N$ layers are regarded as one inhomogeneous birefringent medium. 


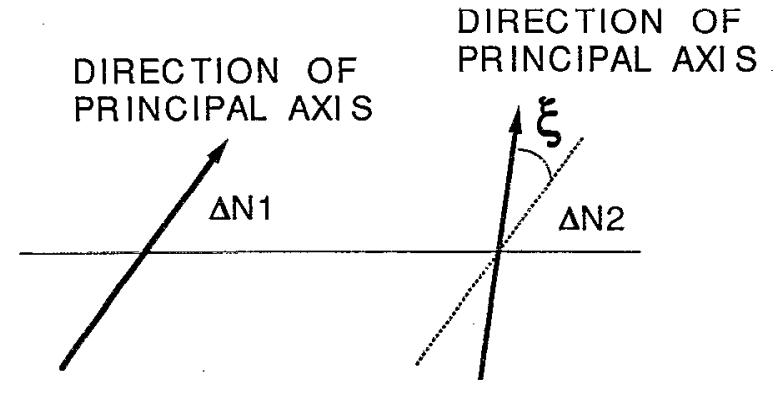

FIG. 3. Birefringence double-layer model. The first and the second layers have retardation of $\Delta N 1$ and $\Delta N 2$, respectively. The angle between principal axes of those layers are $\xi$. Directions of principal axis are shown by arrows.

\section{B. Properties of birefringent multilayer}

The deviation from conventional birefringence becomes evident for large retardations $\delta_{n}$ as can be seen in Eq. (A2) in Appendix A. As a simple example, consider a pair of $\lambda / 4$ plates with an optical axis direction different from each other by $\theta$, i.e., $N=2$ in Eq. (1) and $\delta 1=\delta 2=\pi / 2$. The corresponding operator is $\mathbf{R}(\theta+\xi) \mathbf{P} \pi / 2 \mathbf{R}(-\theta-\xi) \mathbf{R}(\theta) \mathbf{P} \pi / 2 \mathbf{R}(-\theta)$ $=\mathbf{R}(2 \xi)$, which shows that these plates act as a pure rotator.

When a birefringent multilayer medium has a mirror inversion symmetry along the direction of beam propagation, the rotation bias disappears similarly to the case where optical activity is inhibited in molecules having inversion symmetry. An example is polarization of a normally incident beam on a substrate with the opposite side coated with a metallic film as in magneto-optical disks. In this case, Eq. (1) can be expressed by the following form

$$
\begin{aligned}
& \prod_{n=1}^{N} \mathbf{R}\left(\theta_{n}\right) \mathbf{P} \delta_{n} \mathbf{R}\left(-\theta_{n}\right) R l \prod_{n=N}^{1} \mathbf{R}\left(\theta_{n}\right) \mathbf{P} \delta_{n} \mathbf{R}\left(-\theta_{n}\right) \\
& =R l \mathbf{R}(\alpha+\alpha 0) \mathbf{P} 2 \sigma \mathbf{R}(-\alpha-\alpha 0),
\end{aligned}
$$

where $R l$ is the reflectance of the metallic film. Thus, a birefringent multi-layer medium with a mirror inversion symmetry reduces to a conventional birefringent medium. The parameters $\sigma$ and $\alpha$ are those defined by a single pass measurement. A proof is given in Appendix B.

\section{NUMERICAL AND EXPERIMENTAL RESULTS}

In this section we discuss properties of a birefringent multilayer medium based on Eq. (3). Equation (1) with $N=2$ is also used due to its mathematical equivalence to Eq. (3). Figure 3 shows the model and defines the notations $\Delta N 1=\delta_{1} \lambda / \pi, \Delta N 2=\delta_{2} \lambda / \pi$, and $\xi=\theta_{2}-\theta_{1}$, where $\delta_{1}, \delta_{2}$, $\theta_{1}$, and $\theta_{2}$ are those in Eq. (1) for $N=2$ and $\lambda$ is the wavelength. $\Delta N t$ is the effective total retardation $\sigma \lambda / \pi$.

\section{A. Ellipticity of polarization and principal axis of birefringence}

The retardation $2 \Delta$ in a birefringence single layer is often defined by

$$
\Delta=\arctan v\left[\left(I_{\min } / I_{\max }\right)_{\max }\right],
$$

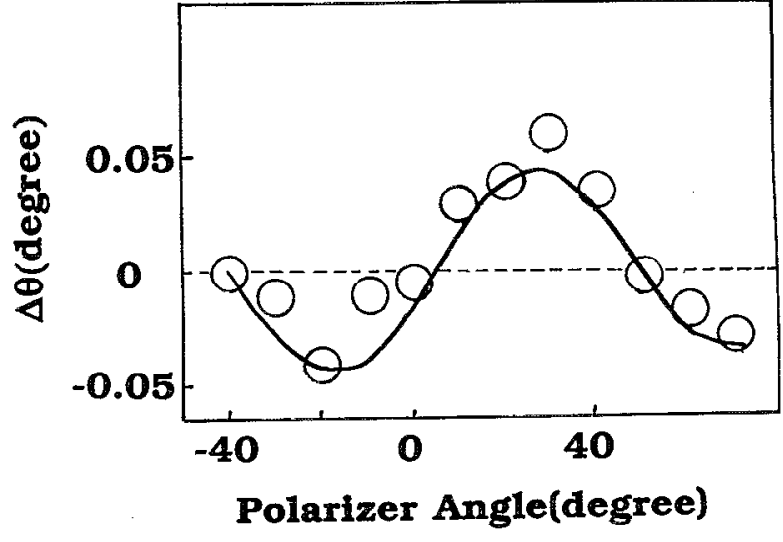

FIG. 4. An example of a relatively conventional behavior of $\Delta \theta$ vs $\mathrm{PO}$ angle variation. A PO angle has a meaning of an angle between a principal axis of retardation and a direction of polarization of an incident beam, when the principal axis of retardations directed at $0^{\circ}$. Open circles and the solid line show data points and calculated results based on a conventional birefringence, respectively. Measured $\Delta N t$ was $8 \mathrm{~nm}$.

where $I_{\min }$ and $I_{\max }$ are the minimum and the maximum power intensities defined by changing the $\mathrm{AO}$ angle at $\mathrm{a}$ given $\mathrm{PO}$ angle. Here, []$_{\max }$ and []$_{\min }$ are defined by changing the $\mathrm{PO}$ angle. In the following, the $\mathrm{PO}$ angle corresponds to the angle between the direction of the electric field of the incident linearly polarized beam and that of the principal axis of retardation. In conventional birefringence the direction of the principal axis is defined as the PO angle at the minimum of $I_{\min } / I_{\max }$.

The ellipticity of a beam passing through a birefringent multilayer medium can be zero at a certain polarization direction, as in conventional birefringence. For a linearly polarized incident beam with $E_{0} x=1$ and $E_{0} y=0$, this occurs at $\alpha=\alpha 0$ in Eq. (3). According to Eq. (3), $I_{\min }$ varies sinusoidally with $\alpha$ with a periodicity of $\pi / 2$. This suggests that $\alpha$ may be regarded as an effective principal axis direction.

\section{B. Rotation of polarization}

The principal polarization direction $\Delta \theta$ of a transmitted beam with respect to that of an incident beam oscillates with $\alpha$ with a periodicity of $\pi / 2$. Unlike $I_{\min }$ vs $\alpha$ this oscillation was not expressed by a simple sinusoidal function (Appendix C). Using Eq. (3), the amplitude of $\Delta \theta$ was found to be governed by the effective total retardation $2 \sigma$, for $\sin \sigma \ll 1$. The $\Delta \theta$ was found to be equal to $\alpha 0$ at $\alpha=\alpha 0$, which is zero for conventional birefringence. That is, the linear polarization rotates by $\alpha 0$ keeping its linearity when the angle betwcen the polarization direction of the incident beam and the effective principal axis is $\alpha 0$.

First, the $\Delta \theta$ variation was measured for a beam passing through a quartz single crystal plate, i.e., a commercial $\lambda / 4$ plate. Periodicity of $\pi / 2$ and symmetry with respect to $\Delta \theta=0$ were clearly observed, and the results agreed well with the conventional model for birefringence. Figure 4 shows how $\Delta \theta$ varies when a beam is transmitted through a polycarbonate plate. The result was close to that for the quartz single crystal platc, but a small asymmetry, or $\Delta \theta$ bias, existed. 


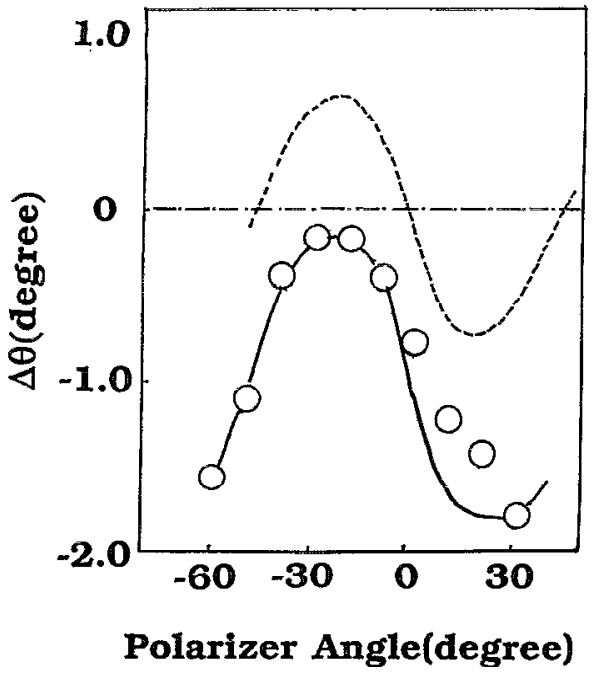

FIG. 5. $\Delta \theta$ vs $\mathrm{PO}$ angle variation in a polycarbonate plate. Open circles are data, and the dashed line and the solid line correspond to the present and conventional models, respectively. Measured $\Delta N t$ was $32 \mathrm{~nm}$.

Furthermore, larger deviations from the conventional model were also observed in other samples. Figures 5 and 6 show measured $\Delta \theta$ variations (circle) together with calculated results based on conventional birefringence (dotted line) and Eq. (3) (solid line). A large $\Delta \theta$ and an asymmetry with respect to $\Delta \theta=0$ is evident in the measured data. It is seen that Eq. (3) fits the data well and satisfies the constraints of $\Delta N t$ and the direction of the effective principal axis. It should be mentioned that these constraints together with $\alpha 0$, which form the complete set of parameters in Eq. (3), are determined experimentally as discussed in Appendix D. In most cases, the magnitude of the $\Delta \theta$ bias was observed to be a few hundredth of a degree.

Figures 7, 8, and 9 show calculated results for typically observed retardation values and illustrate the properties of the present model $(\xi \neq 0)$ as compared with the conventional

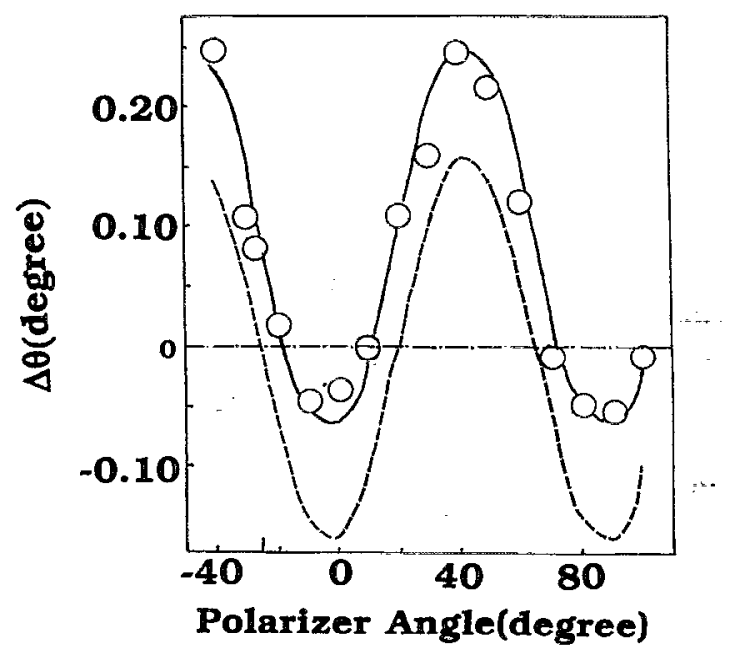

FIG. 6. Same as Fig. 7 in another polycarbonate plate. Measured $\Delta N t$ was $15 \mathrm{~nm}$.

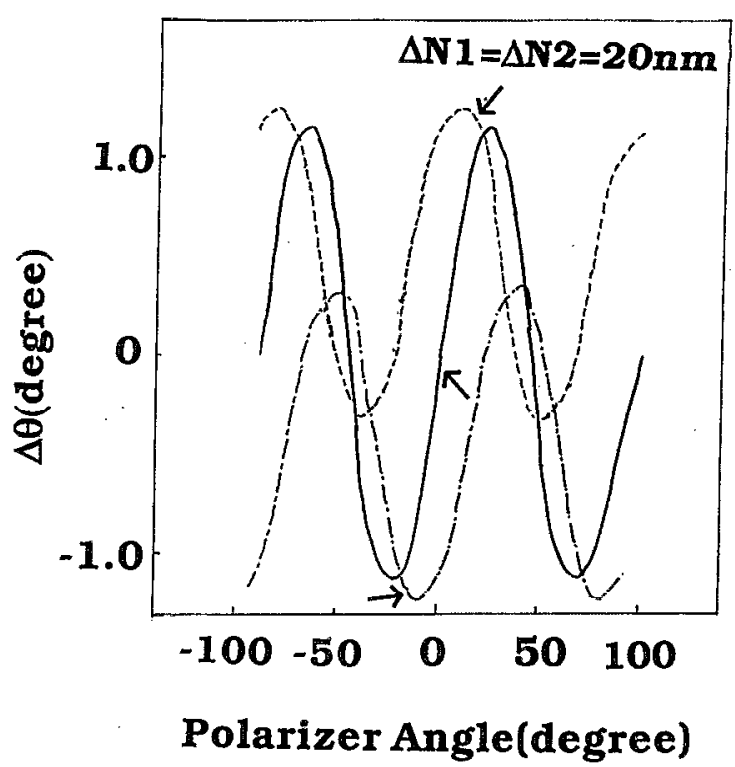

FIG. 7. $\Delta \theta$ vs $\mathrm{PO}$ angle variation calculated for fixed magnitudes of internal retardation $\Delta N 1=\Delta N 2=20 \mathrm{~nm}$ and different $\xi$. The case $\xi=0^{\circ}$ corresponds to conventional birefringence, with a total effective retardation $\Delta N t$ $=\Delta N 1+\Delta N 2$. For $\xi= \pm 30^{\circ}, \Delta N t$ is $32 \mathrm{~nm}$. The dashed line, the dash-dot line, and the solid line correspond to $\xi-30^{\circ}, 0^{\circ},-30^{\circ}$ in the present model, respectively. Arrows indicate the direction of the effective principal axis $\alpha 0$ of each curve.

model $(\xi=0)$. Figure 7 shows $\Delta \theta$ vs $\mathrm{PO}$ angle for tixed internal retardations $\Delta N 1, \Delta N 2$, and different values of $\xi$. Two features are evident. First, the $\Delta \theta$ bias changes sign with the sign of $\xi$. Second, the maximum absolute values of $\Delta \theta$ for $\xi= \pm 30^{\circ}$ are even larger than for $\xi=0^{\circ}$, despite the decrease of $\Delta N t$. Figure 8 also shows $\Delta \theta$ vs PO angle for fixed $\Delta N 1$, $\Delta N 2$, and different $\xi$ 's. With increasing $\xi$, the amplitude of the $\Delta \theta$ oscillation decreases and the asymmetry increases. As

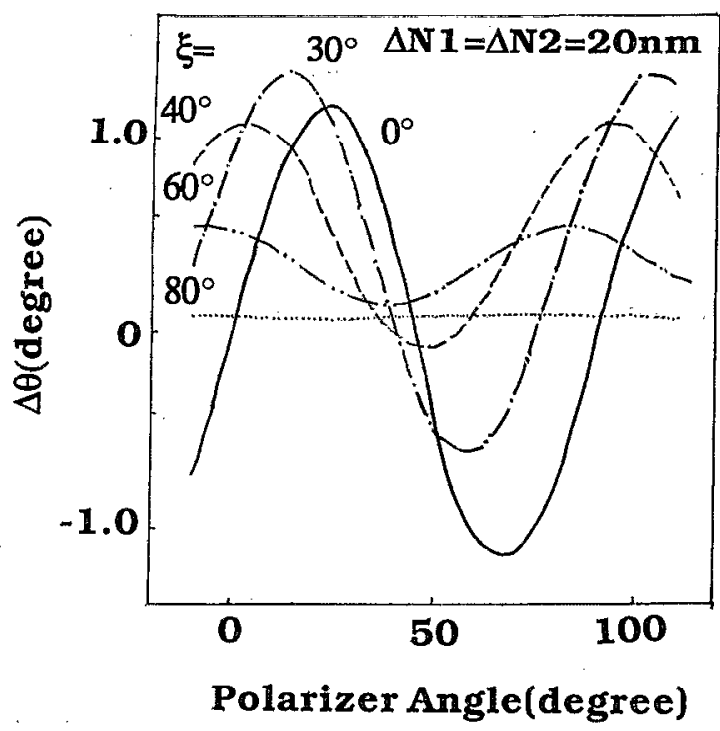

FIO. 8. $\Delta \theta$ vs PO angle variation calculated for fixed magnitudes of internal retardation and different $\xi$. The solid line, the dashed line, the dash dot line; the dash-dot-dot line correspond to $\xi=0^{\circ}, 30^{\circ}, 40^{\circ}, 60^{\circ}, 80^{\circ}$ in the present model, respectively. 


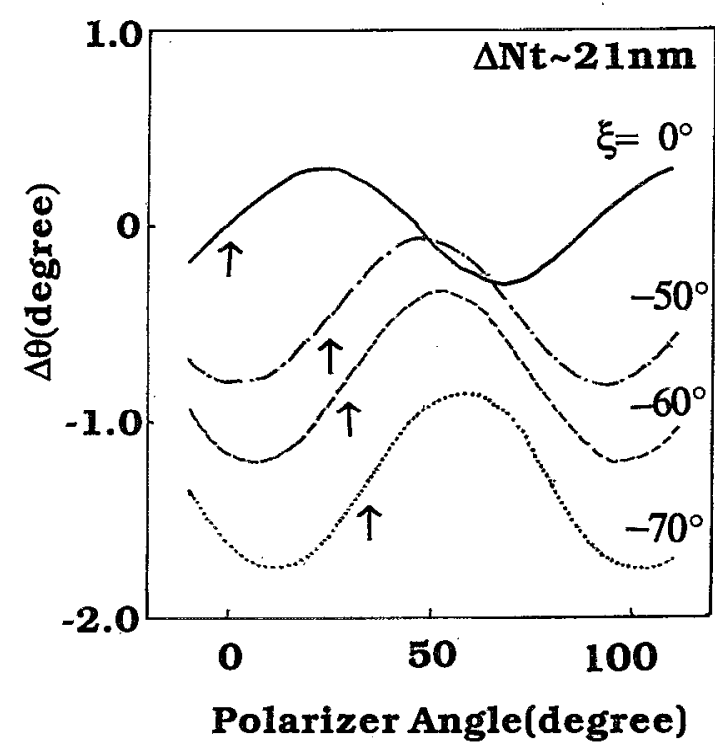

FIG. 9. $\Delta \theta$ vs PO angle variation calculated for a fixed magnitude of an effective total retardation and different $\xi$. The solid line, the dash-dot line the dashed line, the dotted line correspond to $\xi=0^{\circ},-50^{\circ},-60^{\circ}, 70^{\circ}$ in the present model, respectively. Arrows indicate the direction of the effective principal axis $\alpha 0$ of each curve.

discussed above, the decrease of the amplitude is mainly related to that of $\Delta N t$.

Figure 9 shows $\Delta \theta$ vs $\mathrm{PO}$ angle for a fixed $\Delta N t$ and different $\xi$ 's. In this case, the $\Delta \theta$ bias increases with $\xi$. The increase of the $\Delta \theta$ bias resulted mainly from the increase of $\Delta N 1$ and $\Delta N 2$. As expected, the amplitudes of the $\Delta \theta$ oscillations are almost identical since $\Delta N t$ is the same. It is important to note that even for a very small effective total retardation $\Delta N t$ of $21 \mathrm{~nm}$, a rotation of $2^{\circ}$ is possible.

\section{Leak light at extinction angle}

So far, we have discussed the birefringence inhomogeneity along the direction of thickness, since it exhibits properties qualitatively different from the conventional birefringence. In this paragraph we discuss the effect of in-plane inhomogeneity. The major effect of in-plane inhomogeneity of birefringence is to smear out the above variation in $\Delta \theta$.

The retardation $2 \Delta$ is measured by the commonly used ellipticity measurement method mentioned above. The overall variation of $I_{\min }$ with the PO angle for a beam passing through a polycarbonate plate was fitted well by both Eq. (3) and the conventional birefringence model [Eq. (2)]. Here, $I_{\min }$ is the minimum leak power at a given $\mathrm{PO}$ angle as defined before:

Figure 10 shows a closer examination of the $I_{\min }$ variation near $\left[I_{\min }\right]_{\min }$ for a beam passing through a polycarbonate plate. $\left[I_{\min }\right]_{\min }$ is the minimum $I_{\min }$ when changing the PO angle. $\left[I_{\min }\right]_{\min }$ or the smallest observed value of the leak light was $70 \mathrm{nW}$ or $3.5 \times 10^{-5}$ of $\left[I_{\max }\right]_{\max }(=$ the maximum $I_{\max }$ when changing the $\mathrm{PO}$ angle). It is worthwhile noticing that in a quartz single crystal $\left[I_{\min }\right]_{\min }$ was also observed to be $20 \mathrm{nW}$ or $1 \times 10^{-5}$ of $\left[I_{\max }\right]_{\max }$. On the other hand, according to the birefringence multilayer model Eq. (3) and the

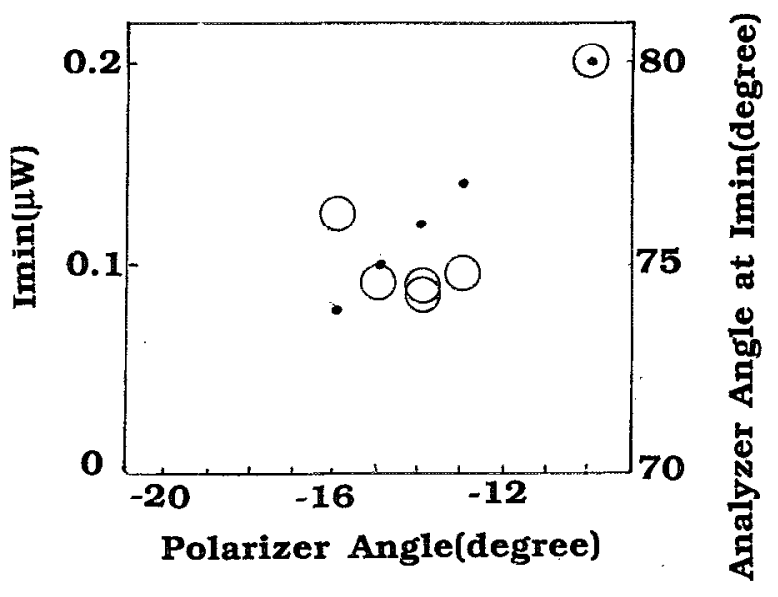

FIG. 10. An example of $I_{\min }$ vs PO angle variation typically observed in polycarbonate plate. $I_{\text {mins }}$ (open circles) are the minimum leak power obtained by scanning $\mathrm{AO}$ angles for a given $\mathrm{PO}$ angle. Dots are $\mathrm{AO}$ angles at $I_{\min }$.

conventional model Eq. (2) $\left[I_{\min }\right]_{\min }$ is always zero. A possible cause of the large experimental $\left[I_{\min }\right]_{\min }$ could be a variation of the parameters in Eq. (3) within the beam spot of $1 \mathrm{~mm}$ in diameter. For example, an in-plane deviation of the principal axis direction of $1^{\circ}$ causes a leak light of $\left[I_{\min }\right]_{\min }=\sin ^{2} \sigma \sin ^{2}\left(2^{\circ}\right)$. This is about $5 \times 10^{-5}$ of $\left[I_{\max }\right]_{\max }$ for a retardation $(\Delta N t)$ of $20 \mathrm{~nm}$. Similar measurements were done using an optical microscope with a long-focus objective lens. $\left[I_{\mathrm{min}}\right]_{\min }$ was still large in a beam spot of less than $0.3 \mathrm{~mm}$ in diameter. Thus, the in-plane inhomogeneity of the birefringence below the submillimeter level is thought to coexist with that along the direction of thickness, which was modeled using the birefringent multilayer approach.

\section{Physical foundation of Eq. (3): Transition region}

In plastics the boundary between adjacent layers is unlikely to be abrupt but changes gradually as in Fig. 11(b). At such a transition region, the Jones matrix Eq. (2) is not applicable.

Generally, an inhomogeneous birefringence may be regarded as an arbitrary stack of two components, i.e., a homogeneous layer expressed by Eq. (2) and a transition layer which contains a change in magnitude of the retardation and a change in the direction of the principal axes. The change in the magnitude is well approximated by Eq. (2). The regions where Eq. (2) are applicable are indicated in Fig. 11(b) by the horizontal bars, and the transition regions, where applicability of Eq. (2) is not certain, are indicated by oblique bars.

A product of the matrices expressed by Eq. (3) is also expressed by Eq. (3) as can be seen from the discussion in Appendix A. Therefore, Eq. (3) is generally valid for an inhomogeneous birefringence, if the transition layer, in which the direction of principal axis changes gradually, is expressed by Eq. (3). This is shown by introducing a local variation of the dielectric tensor in Maxwell equations as 


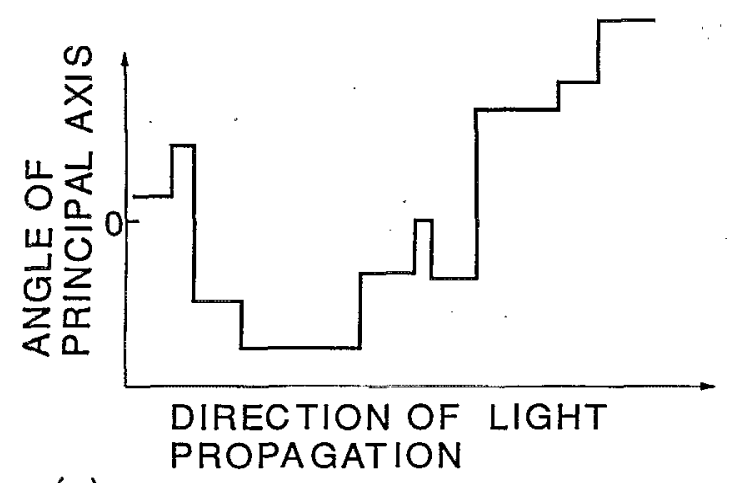

(a)

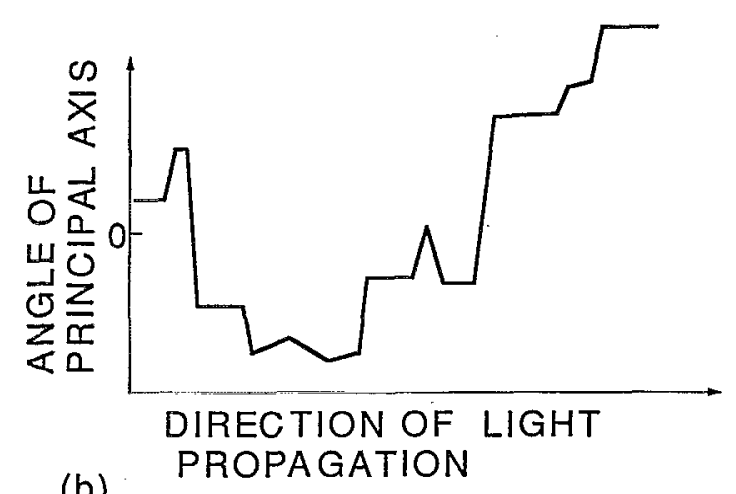

(b)

FIG. 11. Schematic representation of inhomogeneous birefringence showing a random variation of direction of the principal axis (b). Modeling of the variation in (b) by a birefringent multi-layer (a). Horizontal bars indicate the direction the principal axis are approximated to be constant so that a conventional model is applied in this region.

$$
\begin{aligned}
\epsilon(z)=\epsilon 0+\epsilon^{\prime}(z) & =\epsilon 0+\Delta \epsilon \mathbf{R}[\theta(z)]\left(\begin{array}{cc}
1 & 0 \\
0 & -1
\end{array}\right) \mathbf{R}[-\theta(z)] \\
& =\epsilon 0+\Delta \epsilon \mathbf{S}[2 \theta(z)],
\end{aligned}
$$

where $\epsilon 0$ and $\Delta \epsilon$ are constants. Here, we only consider a local vatiation of the principal axis as expressed by $\theta(z)$ and can therefore set $\Delta \epsilon$ to be constant. Furthermore, we assume a constant magnetic permeability $\mu$. In the case of $\mathbf{E}$ being a function of only $z$ and $t$ and $E z$ being zero, the Maxwell equations in c.g.s. units reduce to

$$
\frac{\partial^{2} \mathbf{E}(z, t)}{\partial z^{2}}=\frac{\mu \epsilon \partial^{2} \mathbf{E}(z, t)}{c^{2} \partial t^{2}} .
$$

We introduce a wave function $\Psi^{ \pm}$defined as

$$
\Psi^{ \pm}(z, t)=E x(z, t) \pm i E y(z, t) \text {. }
$$

Using Eqs. (5) and (6) gives

$$
\frac{\partial^{2} \Psi^{ \pm}}{\partial z^{2}}(z, t)=\frac{\mu \epsilon 0 \partial^{2} \Psi^{ \pm}}{c^{2} \partial t^{2}}+\mu \Delta \epsilon e^{ \pm 2 i \theta(z)} \frac{\partial^{2} \Psi^{\mp}}{c^{2} \partial t^{2}} .
$$

Equation (7) is the same equation for the beam propagation in cholesteric liquid crystals. ${ }^{6-9}$ Assuming a stationary wave $\Psi^{ \pm}(z, t)=\Psi^{ \pm}(z) e^{-i \omega t}$, Eq. (7) becomes

$$
-{\frac{d^{2} \Psi^{ \pm}}{d z^{2}}(z)}^{2}-\Delta \mathscr{E} c^{ \pm 2 i \theta(z)} \Psi^{\mp}(z)=\mathscr{E} \Psi^{ \pm}(z),
$$

where $\mathscr{E}=\mu \epsilon 0 \omega^{2}=k_{0}^{2}$ and $\Delta \mathscr{E}=\mu \Delta \epsilon \omega^{2}=(\Delta \epsilon / \epsilon 0) k_{0}^{2}$.

Within the transition layer the linear approximation: $2 \theta(z)=\kappa z$ with $|\kappa z|<\pi / 2$ may he used for $\theta(z)$. Assuming an advancing wave given by $\Psi^{+}(z)=a e^{i k 1 z}+b e^{i k 2 z}$ Eq. (8) is solved rigorously. The solution is expressed by a matrix $\mathbf{T}(l)$ which gives the relation between $\mathbf{E}(z=l, t)$ and $\mathbf{E}(z$ $=0, t)$ as $\mathbf{E}(z=l, t)=e^{i \nu l} \mathbf{T}(l) \mathbf{E}(z=0, t)$, where $\nu$ is a real number. In the following, we present approximate solutions for two limits.

In the slow transition limit with $\kappa \ll \kappa_{0}\left[\equiv(\Delta \epsilon / \epsilon 0) k_{0}\right] \ll k_{0}, \mathrm{~T}(l)$ is found to be

$\left[\cos \sigma \mathbf{R}(\theta)+\epsilon 0 \kappa / \Delta \epsilon k_{0} \sin \sigma \mathbf{R}(\theta-\pi / 2)\right.$

$$
\begin{aligned}
& +i\left(1+\kappa / 2 k_{0}\right) \sin \sigma \mathbf{S}(\theta) \\
& \left.+\kappa / 2 k_{0} \sin \sigma \mathbf{S}(\theta-\pi / 2)\right] /\left(1+\kappa / 2 k_{0}\right),
\end{aligned}
$$

where $\sigma=\kappa_{0} l / 2$ and $\theta=\theta(l)$.

Equation (9) has the form of Eq. (3) except for the correction of the last term which is negligible, since the sum of the $\mathbf{R}$ terms is an $\mathbf{R}$ term itself. By rotating the $x y$ coordinates by an angle $\alpha$, an expression for an arbitrary choice of $x y$ coordinates is obtained. The leading term is

$$
\cos \sigma \mathbf{R}(\theta)+i \sin \sigma \mathbf{S}(\theta+2 \alpha) .
$$

The condition $\kappa \ll \kappa_{0}$ assures $\theta \ll \sigma$. Therefore, Eq. (9) gives only a small rotation bias $\theta$ for $\sigma \leqslant 0$.

In the fast transition limit with $\kappa_{0} \ll \kappa \ll k_{0}, \mathbf{T}(l)$ is given as

$$
\begin{gathered}
\mathbf{R}(-\eta l)+3 i \kappa_{0} / 2 \kappa \sin (\theta+\eta l) \mathbf{S}(\theta) \\
\left.+\kappa_{0} / 2 \kappa \sin [\theta+\eta l) \mathbf{S}(\pi / 2-\theta)\right],
\end{gathered}
$$

where $\eta=-\kappa_{0}^{2} / 2 \kappa$ and $\theta=\theta(l)$.

Averaging the high frequency oscillations, we obtain

$$
\mathbf{R}(-\eta l)+3 i \kappa_{0} / 4 \kappa \cdot \mathbf{S}(\pi / 2-\eta l)+\kappa_{0} / 4 \kappa \mathbf{S}(-\eta l) .
$$

Since $|\kappa l|<\pi / 2$ in the transition layer, Eq. (11) is roughly equal to

$$
\mathbf{I}+3 i \kappa_{0} / 4 \kappa \mathbf{S}(\pi / 2),
$$

which has the same form as Eq. (2). Thus, Eq. (3) is valid beyond a birefringent multilayer model.

Both Eqs. (9) and (10) give Eq. (3) with $a 0<\sigma$ in the transition layer, while birefringence in homogeneous layers is expressed by Eq. (2). Using the same arguments as in Appendix A, the total rotation bias is shown to be small, i.e., of the order of square of the total retardation, as long as $\sigma<1 / N$ in Eqs. (9) and (10) and $\delta_{n} \ll 1 / N[N$ is defined in Eq. (1)]. This means that Eq. (2) is applicable to most plastic substrates for MO disks as long as the prediction of the small rotation bias is not important.

Using similar calculations, we can show that bircfringence can produce an optical rotatory power ${ }^{10.11}$ in the fast transition limit for $|\kappa l| \gg 1 .^{6}$ Therefore, the rotation bias in Eq. (3) in an inhomogeneous birefringent medium can be regarded as a precursory phenomena of an optical activity produced by birefringence. 


\section{A. Comparison with optical rotatory power having birefringence}

Although basic equations for a medium having optical rotatory power and birefringence are dissimilar to Eq. (8), a Jones matrix derived from these equations is shown to be similar to Eq. (3). A Jones matrix for $\epsilon=\epsilon 0+\left(\begin{array}{cc}\Delta \epsilon & -i G \epsilon] \\ -i G \epsilon 0 & \Delta \epsilon\end{array}\right)$ with $G \ll 1$ in a material with a thickness $l$ is

$$
\left(\begin{array}{cc}
e^{i \phi}+k^{2} e^{-i \phi} & -i k\left(e^{i \phi}-e^{-i \phi}\right) \\
i k\left(e^{i \phi}-e^{-i \phi}\right) & e^{-i \phi}+k^{2} e^{i \phi}
\end{array}\right) /\left(1+k^{2}\right),
$$

where $k=G /\left[|\Delta \epsilon|+\sqrt{ }\left(\Delta \epsilon^{2}+G^{2}\right)\right]$ and $\phi=\pi l \sqrt{ }\left[\left(\Delta \epsilon^{2}+G^{2}\right) /\right.$ $\epsilon 0] / \lambda .{ }^{12}$ A nonzero $G$ indicates existence of an optical activity. Equation (12) can also be written as

$$
\cos \beta \mathbf{R}(\alpha 0)+i \sin \beta \mathbf{S}(0),
$$

where $\beta=\tan ^{-1}\left\{\left(1-k^{2}\right) \sin \phi / \sqrt{ }\left[\left(1+k^{2}\right)-\left(1-k^{2} \sin ^{2} \phi\right)\right]\right\}$ and $\alpha 0=\tan ^{-1}\left[2 k /\left(1+k^{2}\right) \tan \phi\right]$. By rotating the coordinates by $\mathbf{R}(\alpha)$, Eq. (13) becomes equal to Eq. (3).

\section{B. Origin of inhomogeneity}

An observation supporting the inhomogeneous birefringence model was recently provided by examining the cross sections of polycarbonate substrates where the retardation near the surfaces was found to be larger than the retardation at the center. ${ }^{13}$ This structure was also suggested by numerical simulations analyzing stress in a polymer resulting from the flow and the cooling process during injection molding. ${ }^{14,15}$ Such inhomogeneity of the refractive index, i.e., birefringence, is related to the local stress variation.

\section{Practical implication for magneto-optical recording}

Birefringence is known to cause noise in the read-back signal of magneto-optical (MO) disks which is usually suppressed by the differential detection system. The noise in polycarbonate MO disks was well accounted for by the conventional model Eq. (2). ${ }^{5}$ However, we have also observed envelope modulation which was due to a rotation of polarization in the substrate. ${ }^{3,5}$ Although its effect can be removed using a high pass filter, this modulation may induce high frequency noise by disturbing the output balance of photodetectors in the differential detection system. The envelope modulation can only be qualitatively explained by Eq. (2). On the other hand, if the direction of the effective principal axis in Eq. (3) changes along a track of a disk, a large envelope modulation can happen even for a very-small retardation. Bccausc of disappearance of the rotation bias due to inversion symmetry as discussed before, this mechanism of envelope modulation does not even occur for a converging beam, as long as the parameters in Eq. (3) are constant in the beam spot. However, it should be noted that the pass of the reflected beam is different from that of the incident beam in a converging beam. This may cause a break of the inversion symmetry leading to envelope modulation, if the disk is tilted or the parameters in the beam spot are not constant. The latter is the in-plane inhomogeneity proposed above.
Indeed, the envelope modulation was significantly reduced when annealed polycarbonate disks were used as substrates for MO disks. ${ }^{3}$ Since annealing releases residual stresses and, in turn, reduces the birefringence inhomogeneity, this experiment supports the above mechanism. The low frequency envelope modulations, which were much larger than predicted by Eq. (2), were also observed in MO disks with other plastic substrates such as epoxy ${ }^{16}$ These observations imply that similar phenomena also exist in other plastics.

It should be mentioned that Eq. (1) can be applied to the total system consisting of an optical head and a MO disk. Therefore, the discussion of the rotation bias in this article also holds for the total system. Obviously, the birefringence of the optical head should be much less than that of the MO disk substrate in order to avoid envelope modulation due to the rotation bias effect.

For a converging beam it is necessary to use a refractive ellipsoid for a birefringence single layer to calculate the refractive indexes for a beam with an arbitrary angle of incidence. We have not generalized Eq. (3) for a beam incident from an arbitrary direction. In this case, the equivalence of Eq. (1) for $N=2$ with Eq. (3) may be used. That is, calculating first the Jones matrices corresponding to each layer for an arbitrary angle of incidence, the net effect for the angle of incidence may be approximated by the product of these two matrices.

\section{CONCLUSION}

Rotation of polarization in polycarbonate plates was measured with high accuracy using a parallel beam and was found to be different from predictions using the conventional model for birefringence [Eq. (2)]. We attributed this result to a birefringence inhomogeneity in the direction of the thickness and proposed a simple formula Eq. (3) as a mathematical model, which is equivalent to birefringence coexisting with optical activity. This proposed formula includes an additional parameter representing a rotation bias which was estimated to be small for a weak birefringence. Mirror inversion symmetry in the direction of propagation reduces Eq. (3) to Eq. (2). For weak birefringence it was shown that an inhomogeneous birefringence has properties similar to the conventional model except for a rotation of polarization. As an application the typically observed low frequency noise in the read-back signal of magneto-optical disks was discussed. It may be worthwhile to note that similar phenomena should be observed in mixtures of randomly oriented anisotropic dielectric or ferroelectric materials such as $\mathrm{BaTiO}_{3}$, $(\mathrm{Pb}, \mathrm{La}) \mathrm{TiO}_{3}$, and $(\mathrm{Pb}, \mathrm{La})(\mathrm{Zr}, \mathrm{Ti}) \mathrm{O}_{3}$. The birefringence in these mixtures having randomly oriented multiple domains should also show the rotation bias effect represented by Eq. (3).

\section{ACKNOWLEDGMENT}

The author would like to acknowledge Dr. Burkhard Friedrichs for useful discussions and Dr. S. Uchida for his encouragement. 
It is useful to derive Eq. (3) using the peculiarity of Eq.

(2) so that the order of magnitude of the parameters in Eq.

(3) can be estimated from those in Eq. (1). Using Eq. (2), Eq.

(1) is

$$
\mathbf{M}=\prod_{n=1}^{N}\left[\cos \delta_{n} \mathbf{I}+i \sin \delta_{n} \mathbf{S}(2 \theta)_{n}\right]
$$

By expanding Eq. (A1) we obtain

$$
\begin{aligned}
& \mathbf{M}=\left(\prod_{n=1}^{N} \cos \delta_{n}\right) \mathbf{I}+i \sum_{n=1}^{N}\left(\sin \delta_{n} \prod_{j \neq n}^{N} \cos \delta_{j}\right) \mathbf{S}\left(2 \theta_{n}\right)-\sum_{m<n}^{N}\left(\sin \delta_{m} \sin \delta_{n} \prod_{j \neq m, n}^{N} \cos \delta_{j}\right) \mathbf{R}\left(2 \theta_{m}-2 \theta_{n}\right) \\
& \text {..... } \\
& +(i)^{2 k-1} \sum_{l_{1}<l_{2} \ldots<l_{k}}^{N}\left(\sin \delta_{l_{1}} \sin \delta_{l_{2}} \ldots \sin \delta_{l_{k}} \prod_{\substack{j \neq l_{1}, l_{2}, \cdots, l_{k} \\
N}}^{N} \cos \delta_{j}\right) \mathbf{S}\left(\sum_{n=k}^{1}(-1)^{n-k_{2}} \theta_{n}\right) \\
& +(i)^{2 k} \sum_{l_{1}<l_{2} \ldots \ldots<l_{k}<l_{k+1}}^{N}\left(\sin \delta_{l_{1}} \sin \delta_{l_{2}} \ldots \sin \delta_{l_{k+1}} \prod_{j \neq l_{1}, l_{2}, l_{k+1}}^{N} \cos \delta_{j}\right) \mathbf{R}\left(\sum_{n=k+1}^{1}(-1)^{n-k-1} 2 \theta_{n}\right)
\end{aligned}
$$

General forms are shown by the last two terms in Eq. (A2). Sums of $\mathbf{S}$ and $\mathbf{R}$ terms can be written as

$$
\begin{aligned}
& \left(\begin{array}{cc}
A & B \\
B & -A
\end{array}\right)=a \mathbf{S}(2 \alpha) \text { for a sum of } \mathbf{S} \text { terms, } \\
& \left(\begin{array}{cc}
C & -D \\
D & C
\end{array}\right)=b^{\prime} \mathbf{R}(2 \beta) \text { for a sum of } \mathbf{R} \text { terms and }
\end{aligned}
$$$$
\left(\prod_{n=1}^{N} \cos \delta_{n}\right) \mathbf{I}=c \mathbf{I} \text { for } \mathbf{I} \text { term, }
$$

$b^{\prime} \mathbf{R}(2 \beta)+c \mathbf{I}=b \mathbf{R}(2 \alpha 0)$,

where $A, B, C, c$, and $D$ are real numbers, and $a=\sqrt{ }\left(A^{2}+B^{2}\right), \quad b^{\prime}=\sqrt{ }\left(C^{2}+D^{2}\right), \quad b=\sqrt{ }\left[(c+C)^{2}+D^{2}\right], 2 \alpha$ $=\arctan (B / A), 2 \beta=\arctan (D / C)$, and $2 \alpha 0=\arctan [D /(c$ $+C)]$. Because of the unitarity of $\mathbf{M}:[b \mathbf{R}(2 \alpha 0)$ $+i a \mathbf{S}(2 \alpha)]+[b \mathbf{R}(2 \alpha 0)+i a \mathbf{S}(2 \alpha)]=\mathbf{I}, a^{2}+b^{2}=1$. Thus, we can choose $a=\sin \sigma, b=\cos \sigma$, and $\mathrm{M}$ is written as

$$
\begin{aligned}
M & =c \mathbf{I}+i a \mathbf{S}(2 \alpha)+b^{\prime} \mathbf{R}(2 \beta) \\
& =\cos \sigma \mathbf{R}(2 \alpha 0)+i \sin \sigma \mathbf{S}(2 \alpha) .
\end{aligned}
$$

By the way, Eq. (A2) for $N=2$ is $\cos \delta_{1} \cos \delta_{2} \mathbf{I}-\sin \delta_{1} \sin \delta_{2} \mathbf{R}\left(2 \theta_{1}-2 \theta_{2}\right)$

$+i\left[\sin \delta_{1} \cos \delta_{2} \mathbf{S}\left(2 \theta_{1}\right)+\sin \delta_{2} \cos \delta_{1} \mathbf{S}\left(2 \theta_{2}\right)\right]$.

Equation (A4) has the same form as Eq. (3) and three free parameters. Therefore, Eq. (3) is expressed by Eq. (A4).

In most cases, $b^{\prime}$ (or $\delta_{n}$ ) $\ll 1$ and thus the third term $\mathbf{R}$ in Eq. (A3) can be neglected in the first order in $b^{\prime}$. Then, Eq. (A3) represents the conventional birefringence by a single layer. The $b^{\prime} \mathbf{R}(2 \beta)$ represents the rotation bias due to the inhomogeneity.

The order of magnitude of each term in in Eq. (3) can be evaluated using Eq. (A2). Noting that retardation $\delta$ is proportional to thickness, we evaluate the order of $\delta_{n}$ as $\sigma / N$, where $\sigma(<1)$ is the effective total retardation in Eq. (3). The orders of $c, a$, and $b^{\prime}$ in Eq. (A3) are 1 , $\sigma\left(1+\sigma^{2}+\sigma^{4}+\sigma^{6} \ldots . ..\right)$, and $\sigma^{2}\left(1+\sigma^{2}+\sigma^{4}+\sigma^{6} \ldots \ldots\right)$, respectively. Therefore, Eq. (A3) can be regarded as Eq. (2) with a small modification R. Especially, for $\delta_{n} \ll 1 / N$ (e.g., $\left.\delta_{n}<1 / 5 N\right), b^{\prime}$ is an order of $c^{2}$.

\section{APPENDIX B: PROOF OF DISAPPEARANCE OF $\mathbf{R}$ TERMS IN EQ. (4)}

$$
\begin{aligned}
& \prod_{n=1}^{N} \mathbf{R}\left(\theta_{n}\right) \mathbf{P} \delta_{n} \mathbf{R}\left(-\theta_{n}\right) R l \prod_{n=N}^{1} \mathbf{R}\left(\theta_{n}\right) \mathbf{P} \delta_{n} \mathbf{R}\left(-\theta_{n}\right) \\
& \quad=R l\left(\prod_{n=1}^{N-1} \mathbf{R}\left(\theta_{n}\right) \mathbf{P} \delta_{n} \mathbf{R}\left(-\theta_{n}\right)\right) \mathbf{R}\left(\theta_{N}\right) \mathbf{P} 2 \delta_{N} \mathbf{R}\left(-\theta_{N}\right)\left(\prod_{n=N-1}^{1} \mathbf{R}\left(\theta_{n}\right) \mathbf{P} \delta_{n} \mathbf{R}\left(-\theta_{n}\right)\right)
\end{aligned}
$$




$$
\begin{aligned}
= & R l \prod_{n=1}^{N-1} \mathbf{R}\left(\theta_{n}\right) \mathbf{P} \delta_{n} \mathbf{R}\left(-\theta_{n}\right)\left[\cos 2 \delta_{N} \mathbf{I}+i \sin 2 \delta_{N} \mathbf{S}\left(2 \theta_{N}\right)\right] \prod_{n=N-1}^{1} \mathbf{R}\left(\theta_{n}\right) \mathbf{P} \delta_{n} \mathbf{R}\left(-\theta_{n}\right) \\
= & R l \prod_{n=1}^{N-2} \mathbf{R}\left(\theta_{n}\right) \mathbf{P} \delta_{n} \mathbf{R}\left(-\theta_{n}\right)\left[\left\{\cos ^{2} \delta_{N-1} \cos 2 \delta_{N}-\sin 2 \delta_{N-1} \sin 2 \delta_{N} \cos \left(2 \theta_{N}-2 \theta_{N-1}\right)-\sin ^{2} \delta_{N-1} \cos 2 \delta_{N}\right\} \mathbf{I}\right. \\
& \left.+i\left\{\sin 2 \delta_{N-1} \cos 2 \delta_{N} \mathbf{S}\left(2 \theta_{N-1}\right)+\cos ^{2} \delta_{N-1} \sin 2 \delta_{N} \mathbf{S}\left(2 \theta_{N}\right)-\sin \delta_{N-1} \sin 2 \delta_{N} \mathbf{S}\left(4 \theta_{N-1}-2 \theta_{N}\right)\right\}\right] \\
& \times \prod_{n=N-2}^{1} \mathbf{R}\left(\theta_{n}\right) \mathbf{P} \delta_{n} \mathbf{R}\left(-\theta_{n}\right) .
\end{aligned}
$$

As shown above, the product $\mathbf{R}\left(\theta_{n}\right) \mathbf{P} \delta_{n} \mathbf{R}\left(-\theta_{n}\right) \mathbf{R}\left(\theta_{n}\right) \mathbf{P} \delta_{n} \mathbf{R}\left(-\theta_{n}\right)$ is expressed only by $\mathbf{I}$ and $\mathbf{S}$, and also the product $\mathbf{R}\left(\theta_{n}\right) \mathbf{P} \delta_{n} \mathbf{R}\left(-\theta_{n}\right) \quad \mathbf{S}$ $\mathbf{R}\left(\theta_{n}\right) \mathbf{P} \delta_{n} \mathbf{R}\left(-\theta_{n}\right)$ is expressed only by $\mathbf{I}$ and $\mathbf{S}$.

By induction, Eq. (B1) contains only $\mathbf{I}$ and $\mathbf{S}$ terms and no $\mathbf{R}$ terms. Equation (B1) contains no $\mathbf{R}$ terms and using Eq. (A3), Eq. (B1) is expressed as

$$
R l[c \mathbf{I}+i \bar{a} \mathbf{S}(2 \alpha+2 \alpha 0)] .
$$

Using the same arguments used to derive Eq. (A3) from Eq. (A2) and choosing $c=\cos 2 \sigma$ and $a=\sin 2 \sigma$, Eq. (B2) is $R l\left[\cos 2 \sigma \mathbf{I}+i \sin 2 \sigma^{*} \mathbf{S}(2 \alpha+2 \alpha 0)\right]$

$$
=R l \mathbf{R}(\alpha+\alpha 0) \mathbf{P} 2 \sigma \mathbf{R}(-\alpha-\alpha 0) .
$$

Eq. (B3) is exactly same as Eq. (2).

The parameters in Eq. (B3) are related to those for birefringence in a single path measurement. Using Fq: (3), the Jones matrices for an incident and a reflected beams are

$$
\begin{aligned}
& \cos \sigma \mathbf{R}(2 \alpha 0)+i \sin \sigma \mathbf{S}(2 \alpha) . \\
& R l[\cos \sigma \mathbf{R}(-2 \alpha 0)+i \sin \sigma \mathbf{S}(2 \alpha)],
\end{aligned}
$$

respectively. The argument of $\mathbf{S}$ and the $\sigma$ in a reflected beam were determined from a consideration on symmetry. The argument of $\mathbf{R}$ in a reflected beam was determined from the requirement that a real part of the product of these terms should be proportional to I as in Eq. (B2). Thus, the product gives Eq. (B3).

\section{APPENDIX C: CALCULATION OF $\Delta \theta$}

$E x$ and Ey at a same position satisfy an equation for an ellipse. Principal axis of this ellipse is given by diagonalizing the equation. Using Eqs. (1a), (1b), (A3), and notations in Appendix $A$, the following set of equations gives the principal axis direction $(=\Delta \theta)$ with respect to that of an incident beam with $E_{0} x=1, E_{0} y=0$,

$$
\begin{gathered}
\tan \Delta \theta=\left(F \pm \sqrt{ } F^{2}+4\right) / 2 \\
F=-2\left[a^{2} \cos 4 \alpha+\left[(c+C)^{2}-D^{2}\right] /\right. \\
{\left[a^{2} \sin 4 \alpha+2(c+C) D\right] .}
\end{gathered}
$$

For the conventional birefringence, $D=C=0, a=\sin \sigma$, $c=\cos \sigma$, and Eq. (C2) is $F=-2\left(\cot 4 \alpha+\cot ^{2} \sigma \sin 4 \alpha\right)$.
The amplitude of a $\Delta \theta$ oscillation given by Eq. (C1) is estimated from the maximum and the minimum values of $F$ with respect to $\alpha$. For $D^{2} \& a^{2} \&(c+C)^{2}$, this is approximated by, $-2(c+C) /[ \pm a+(c+C) D]$. This shows that the amplitude is mainly determined by $(c+C) / a$, which is roughly equal to $\cot \sigma$.

\section{APPENDIX D: EXPERIMENTAL DETERMINATION OF PARAMETERS IN EQ. (3)}

We use a randomly polarized light source, and set a $P O$ angle $=\phi$ and an $\mathrm{AO}$ angle $=\chi$. Using Eq. (3), the power intensity $I$ is given by

$I=\cos ^{2} \sigma \cos ^{2}(\phi-\chi-2 \alpha 0)+\sin ^{2} \sigma \cos ^{2}(\chi+\phi-2 \alpha)$,

where $\alpha 0=\tan ^{-1}[D /(c+C)] / 2$ as in Appendix $\Lambda$. For $\phi-\chi$ $-2 \alpha 0= \pm \pi / 2$ and $\chi+\phi-2 \alpha= \pm \pi / 2, I=0$. Thus, a set of $\chi$, $\phi$ for $I=0$ gives $\alpha$ and $\alpha 0$. When $\phi$ and $\chi$ are changed by $\Delta \phi$ from the values for $I=0, I=\sin ^{2} \sigma \cos ^{2}(2 \Delta \phi)$. The coefficient of $\cos ^{2}(2 \Delta \phi)$ gives $\sin ^{2} \sigma$. When a PO and an AO angle are precisely measured, these three values can determine three free parameters in Eq. (3). In addition, $\alpha 0$ and ellipticity can be used to determine the parameters.

${ }^{1}$ N. Imamura, S. Tanaka, F. Tanaka, and Y. Nagao, IEEE Trans. Mag. MAG-21, 1607 (1985).

${ }^{2}$ Y. Watanabe, J. Sasaki, Y. Kobayashi, and T. Yoshitomi, IEEE Trans. Mag. MAG-23, 2623 (1987).

${ }^{3}$ S. Fukunishi and I. Hatakeyma, Jpn. J. Appl. Phys. 26(Suppl. 26-4) 91 (1987).

${ }^{4}$ For example, M. Born and E. Wolf, Principles of Optics, 6th ed. (Pergamon, Oxford, 1980), ISBN 0-08-026481-6, p. 695.

${ }^{5}$ Y. Watanabe, A. Ohya, and M. Komatsu, J. Magn. Soc. Jpn. 11 (Suppl. S1), 313 (1987).

${ }^{6} \mathrm{P}$. G. de Gennes, The Physics of Liquid Crystals (Clarendon, Oxford, 1974), ISBN 0-19-851285-6, p. 228.

${ }^{7}$ C. Mauguin, Bull. Soc. Fr. Miner. Crystallogr. 34, 3 (1911).

${ }^{8}$ C. W. Oseen, Trans. Faraday Soc. 29, 833 (1933).

${ }^{9} \mathrm{H}$. De Vrices, Acta Crystallogr. 4, 219 (1951).

${ }^{10} \mathrm{H}$. Eyring, J. Walter, and G. Kimball, Quantum Chemistry (Wiley, International, New York, 1949), p. 342.

${ }^{11}$ L. D. Landau and E. M. Lifshitz, Electrodynamics of Continuous Media (Pergamon, Oxford, 1960), (Tokyo-tosho, Tokyo, 1962), p. 83.

${ }^{12}$ For example, T. Tsuruta, Ohyo Kohgaku 2 (Baihukan, Tokyo, 1990), ISBN4-563-02332-9 C3342, pp. 174-191 (in Japanese).

${ }^{13}$ For example, R. Wimberger-Friedel, Polym. Eng. Sci. 30, 813 (1990).

${ }^{14}$ A. A. Flaman, Polym. Eng. Sci. 33, 193 (1993).

${ }^{15}$ B. Friedrichs (private communication).

${ }^{16} \mathrm{Y}$. Watanabe, A. Ohya, and M. Komatsu (unpublished). 